\section{Tiselius, Arne Wilhelm Kaurin}

\author{
A. M. Gressner ${ }^{1}$ und O. A. Gressner ${ }^{2}$ \\ ${ }^{1}$ Labor Dr. Wisplinghoff Berlin, Berlin, Deutschland \\ ${ }^{2}$ Labor Dr. Wisplinghoff Köln, Köln, Deutschland
}

Lebensdaten Schwedischer Wissenschaftler, geboren am 10. August 1902 in Stockholm, gestorben am 29. Oktober 1971 in Uppsala.

Verdienste Tiselius studierte an der Universität Uppsala Physik, Chemie und Mathematik und wurde anschließend (1925) Assistent im Arbeitskreis von Theodor Svedberg ( $\triangleright$ Svedberg, Theodor; $\triangleright$ Svedberg-Einheit), wo er mit dem Arbeitsgebiet der Proteintrennungsmethodik in Kontakt kam. Die Beschäftigung mit elektrophoretischen Untersuchungen von Proteinen führte im Jahr $1930 \mathrm{zu}$ seiner Promotion mit dem Thema „The moving boundary method for studying the electrophoresis of proteins". Nach einem Forschungsaufenthalt in den USA nahm Tiselius in Uppsala seine elektropho- retischen Forschungsarbeiten wieder auf, die zur Konstruktion der „Tiselius-Elektrophoreseapparatur“ (1937) führten. Apparatur und Methodik erlaubten eine wesentlich schnellere Separation der Proteine, die mit der Schlierenmethode nach August Toepler (1864) sichtbar gemacht und für biochemische Untersuchungen eingesetzt werden konnten. Im Schlierendiagramm beschrieb Tiselius erstmals 5 Banden: - Albumin, $\alpha-1-, \alpha-2-, \beta$ - und $\gamma-\triangleright$ Globuline ( $\triangleright$ Serumprotein-Elektrophorese). Für die in der Folgezeit weiter ausgebaute und verfeinerte Methode, z. B. für die Trennung der humanen Plasmaproteine, erhielt Tiselius zahlreiche Auszeichnungen darunter im Jahr 1948 den Nobelpreis für Chemie, 12 Ehrendoktorate und die Präsidentschaft der Nobel-Stiftung (s. a. $>$ Elektrophorese).

\section{Literatur}

Tiselius A (1937) A new apparatus for electrophoretic analysis of colloidal mixtures. Trans Faraday Soc 33:524-531 